\title{
Study of the Interaction of a Packing Device with a Coal Seam at Interval Hydraulic Fracturing
}

\author{
Vladimir Klishin ${ }^{1}$, Sergei Klishin ${ }^{2,}$, and Alexander Tatsienko ${ }^{3}$ \\ ${ }^{1}$ Institute of Coal, Federal Research Center for Coal and Coal Chemistry, 650065 Kemerovo, Russia \\ ${ }^{2}$ N.A. Chinakal Institute of Mining, 630091 Novosibirsk, Russia \\ ${ }^{3}$ T.F. Gorbachev Kuzbass State Technical University, 650000 Kemerovo, Russia
}

\begin{abstract}
The technological scheme of the interval hydrodynamic influence on the coal seam is considered. The design of a double-sided packer is presented, the results of laboratory tests to determine the axial movement of the seals during the interval fracturing are shown. The numerical scheme based on the Finite Element Method has been developed for calculating the stress-strain state of a rock in the vicinity of a well, loaded by internal pressure through packer seals. The pressure acting on the contacts between the seals and the well is calculated, the stress distribution in the geomaterial is investigated.
\end{abstract}

\section{Introduction}

To increase the productivity of preparatory workings and clean faces of coal mines, as well as to ensure the safety of working conditions by the gas factor, not only reliable methods for the degassing of coal seams are necessary, but special equipment must also be created, which is the subject of wide discussion by the scientific community [1-9].

According to the existing methods of degassing, the radius of impact on the rock mass through a separate degassing well is extremely small. Therefore, it is necessary to expand the impact zone from one well due to new innovative methods of intensifying the process of degassing of coal seams. By analogy with oil extraction technologies, it was proposed to use the method of influence on a coal massif by directed hydraulic separation of the seam from the free surface [4]. Subsequently, this method was used in various versions from underground mine workings through existing degassing wells, which is the subject of numerous theoretical and practical studies [10-18].

\subsection{Interval hydraulic fracturing of coal bed}

The gas-releasing ability of loaded coal seams can be raised by improving their reservoir properties based on the interval hydraulic fracturing method $[10,11]$. New means of implementing this technology have been developed, laboratory studies and the first experimental tests in a coal mine have been performed [17].

The technological scheme for implementing the technology of interval fracturing is as follows. At the initial stage, the dual packer enters the well with its subsequent sealing by supplying the working fluid to the elastically expanding sleeves (seals). With a further 
increase in pressure, a valve works that is installed between the two seals, which leads to the creation of one or more hydraulic fracture cracks. Then the pressure is released. At the last stage, the depressurization of the well and the movement of the dual packer occurs. To implement this technology, a two-sided (dual) packer has been developed. It includes two elastically expanding sleeves with a diameter of $76 \mathrm{~mm}$, between which there is an interpacker valve designed to control the pressure of the working fluid in the hydraulic system of the fracturing device. It serves for timely packing of the well in the area between the elastically expanding seals and a further increase in pressure in this area to a value sufficient for hydraulic fracturing [11]. When the fluid is supplied, the area is sealed by increasing the outer diameter of the elastically expanding packer shells. With a further increase in pressure, the valve is triggered, which leads to the formation of one or more hydraulic fracture cracks. Of particular importance is the design of the interpacker valve, which provides the supply of working fluid to any sealed section of the well. Thus, induced technological fracturing occurs, including due to the opening of existing cracks. The best result, in this case, is the perpendicularity of the hydraulic fracture cracks and the natural system of cracks of the rock massif.

It should be noted that artificially created cracks develop in an energetically favorable direction, which depends on the initial stress state of the massif and its natural fracture. Taking into account that existing bedding cracks (along the strike of the seam) are almost closed due to the effect of rock pressure when implementing hydraulic fracturing technology, the main task is to create cracks oriented across the well (across the strike of the coal seam).

Thus, the difficulties in implementing the technology consist of the creation of directional hydraulic fractures, oriented across the wells drilled from the preparatory workings parallel to the face. This direction of movement of the cracks is the most advantageous from the degassing point of view, and multiple repetitions with a high density of hydraulic fractures in one well allow increasing the area of gas drainage hundreds of times. Under the influence of the internal pressure of the fluid, the opening of artificial cracks increases, as a result of which their relationship with the system of natural cracks not opened by the well expands. During the hydraulic fracturing operation, the created fractures propagate along the path of least resistance perpendicular to the direction of action of the minimal principal stress. It is known that the choice between the occurrence of longitudinal and transverse cracks is determined by the length of the isolated cavity between the packers $L$ and the well diameter $d$ : for $L<2 d$, a single transverse crack forms; when $2 d<L<4 d$, multiple cracks of various types form; at $L>4 d$, a single longitudinal crack is formed [18].

\subsection{Interaction of the seals of the packer and the borehole walls - laboratory tests}

When execution of interval hydraulic fracturing of a coal seam, difficulties arise associated with the deviation of the cross-sections of degassing wells from a circular shape [16], including due to the bending of the rod during drilling [19]. Moreover, the currently available borehole seals, made in the form of elastically expanding sleeves, do not provide reliable operation of the device at high pressures of the working fluid and unstable dimensions of the well (from 93 to $105 \mathrm{~mm}$ ). This is due to the fact that at the time of supplying the working fluid to the device, the elastic elements of the sealants increase in the diametrical direction up to the contact with the walls of the well. In this case, they are shortened in the axial direction. Since the two sealants are rigidly connected to each other through the valve body, significant tensile forces arise in the place of attachment of the elastic elements, tending to break this connection, which reduces the reliability of the device.

To measure the axial displacement of the sealants from the applied internal pressure, laboratory tests were performed in pipes with a diameter of 80 and $105 \mathrm{~mm}$. Figure 1 shows 
a bench test of packer functioning inside a pipe with a diameter of $105 \mathrm{~mm}$. Here is a dual packer with a diameter of $76 \mathrm{~mm}$, a length of $1113 \mathrm{~mm}$ and a rubber part of $830 \mathrm{~mm}$. The packer was opened at a pressure of 0.1 to $0.5 \mathrm{MPa}$, with the packer end face shifted by 80 $\mathrm{mm}$ (Figure 1a). An increase in pressure from 0.5 to $2.0 \mathrm{MPa}$ led to a reliable fastening of the packer, the end of which at the same time moved another $15 \mathrm{~mm}$ (Figure 1b). A further increase in pressure to $5.0 \mathrm{MPa}$ did not lead to a displacement of the packer end face (Figure 1c).
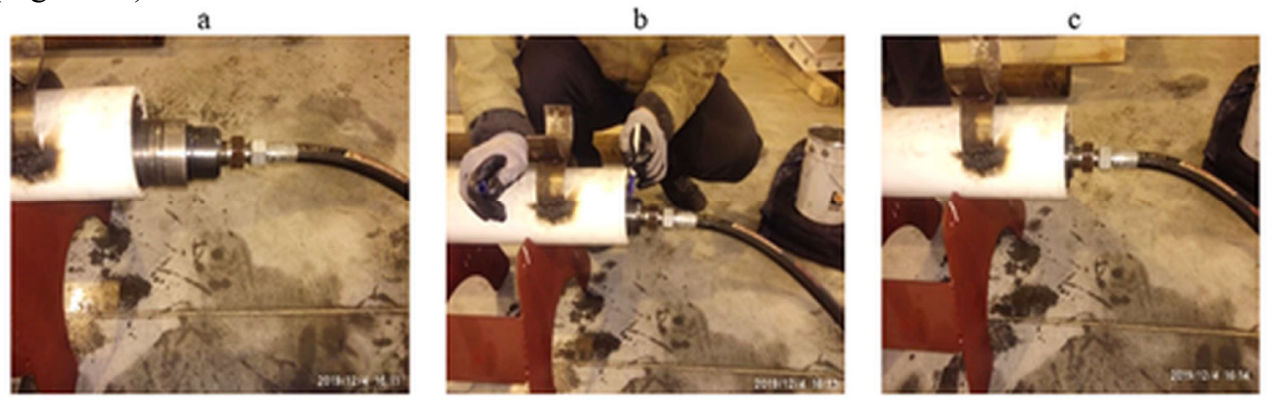

Fig. 1. Bench tests of the seal in a pipe with a diameter of $105 \mathrm{~mm}$.

To improve the reliability the new device design has been proposed by eliminating tensile forces at the point of attachment of the elastic elements to the bushings of the sealants [20].

\section{Numerical study of the stress-strain state of borehole walls loaded with packer seals}

\subsection{Boundary value problem formulation}

Earlier the problem of elastic-plastic deformation of a rock sample during directional hydraulic fracturing was considered and the influence of the working fluid pressure in the inter-package space was shown [21]. In the presented work the problem about loading the borehole walls by packer seals expanding under the influence of internal pressure was numerically solved by the Finite Element Method in the framework of the linear theory of elasticity in a three-dimensional axisymmetric formulation by the COMSOL Multiphysics ${ }^{\circledR}$ software package.

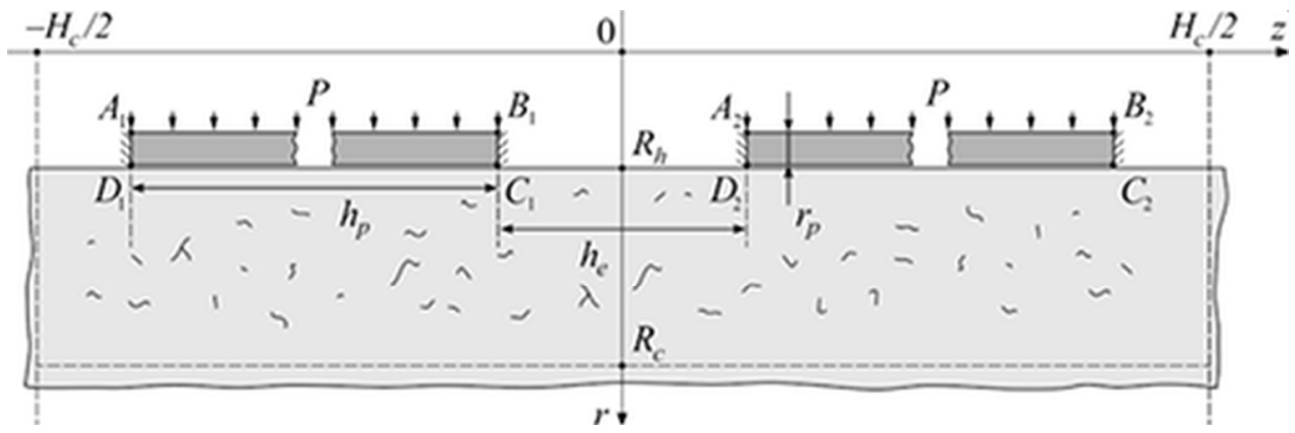

Fig. 2. Scheme of numerical calculation in the Orz plane.

The rock and seals were represented by materials having different elastic coefficients. The loading was carried out by internal pressure applied to the seals without gravity. At the interaction of the seals with the borehole walls, an absolutely rigid contact without slipping 
was considered. Figure 2 shows a section of the studied area by the $O r z$ plane. Here $O z$ is the axis of symmetry in the space $\operatorname{Or} \theta z$.

The dimensions of the studied coal area $H_{c}=2.0 \mathrm{~m}, R_{c}=1.0 \mathrm{~m}$ along the $O z$ and $O r$ axes, respectively; well radius $R_{h}=0.05 \mathrm{~m}$. Two seals of a fixed length $h_{p}=0.5 \mathrm{~m}$ are located symmetrically with respect to the $O r$ axis at a distance $h_{e}$ from each other. Seal thickness $r_{p}=15 \mathrm{~mm}$. To compare the contact interaction, two types of calculations were performed. In the first case, the gap between the seals and the borehole wall was $1 \mathrm{~mm}$, i.e. contact was absent at the initial stage of loading. In the second case, the gap value was zero, i.e. the contact was fully developed from the initial moment of loading. Physical parameters of materials are presented in the Table 1 .

Table 1. Elastic coefficients of materials.

\begin{tabular}{|c|c|c|}
\hline & Coal & Rubber \\
\hline Young's modulus $E$, GPa & 5.4 & 0.5 \\
\hline Poisson's ratio $v$ & 0.16 & 0.45 \\
\hline
\end{tabular}

Let's consider the loading conditions. At the inner boundaries $A_{1} B_{1}$ and $A_{2} B_{2}$ of the seals, a pressure $P$ was set that monotonically increased from 0 to $200 \mathrm{~atm}$ (which corresponds to values of $0 \ldots 20.3 \mathrm{MPa}$ ). The borehole wall $r=R_{h}$ is free of stress at the areas without contact with the seals. At the ends of the seals (surfaces $A_{1} D_{1}, B_{1} C_{1}, A_{2} D_{2}$ and $B_{2} C_{2}$ ), the condition of rigid fastening is specified: $\boldsymbol{u}(r, z) \equiv 0$, where $\boldsymbol{u}(r, z)$ is the displacement vector. On parts of the contour bounding the rock, the condition of rigid fastening is also set:

$$
\begin{aligned}
& \boldsymbol{u}(r, z) \equiv 0 \text { at } r=R_{c} \text { and }-0.5 H_{c} \leq z \leq 0.5 H_{c} \\
& \boldsymbol{u}(r, z) \equiv 0 \text { at } z= \pm H_{c} \text { and } r_{h} \leq r \leq R_{c}
\end{aligned}
$$

\subsection{Results of numerical calculations}

During loading, with an increase in the steps of internal pressure $P$, the packer seals expand, and the area of their contact with the borehole wall increases. A series of calculations was carried out for various values of the distance between the seals $h_{e}=10,20,30,40$, and $50 \mathrm{~cm}$.

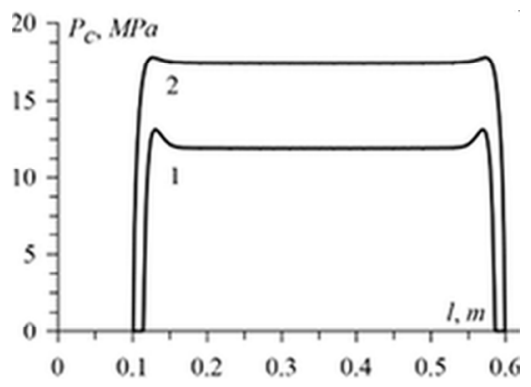

Fig. 3. Pressure plots on the contact between the seal and the borehole wall: 1 - the initial gap between the seal and the borehole well is $1 \mathrm{~mm} ; 2$ - without initial gap.

Pressure plots $P_{c}$ acting on the contact between one of the seals and the borehole wall with a value of $h_{e}=20 \mathrm{~cm}$ are shown on Figure 3. Here, the graph having number 1 corresponds to the initial gap of $1 \mathrm{~mm}$, and the graph having number 2 corresponds to the absence of the initial gap. It can be seen that the maximum pressure is reached at the ending points of the contact surface, and the average pressure is lower than the applied internal 
pressure $P=200 \mathrm{~atm}$. It should be noted that when the distance between the seals (parameter $h_{e}$ ) changes, the pressure plots do not change.

Consider the stress-strain state of the medium in the vicinity of the well at the final stage of loading. Here the following should be noted. It is well known that rocks have different compressive and tensile strengths. Moreover, the tensile strength can be tens times less than the compressive strength. Therefore, we are primarily interested in areas of material where tensile stresses dominate. Calculations showed that tensile stresses in the material arise in the region bounded by seals $\left(-h_{e} / 2<\mathrm{z}<h_{e} / 2\right)$ and adjacent to the well wall. For an example Figure 4 shows the distribution of axial stresses $\sigma_{z}$ for the parameter value $h_{e}=20 \mathrm{~cm}$.
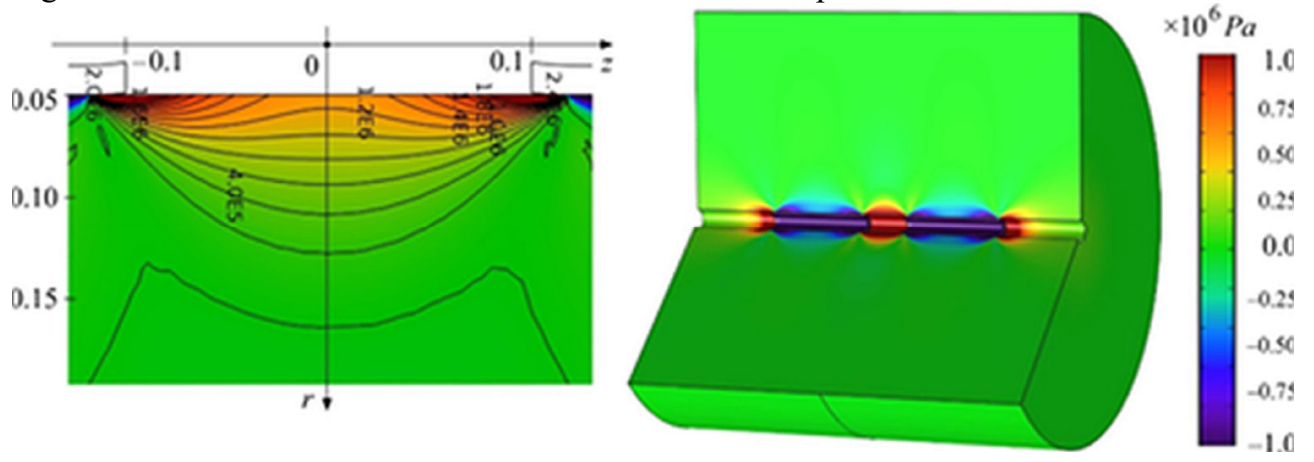

Fig. 4. Axial stress distribution $\sigma_{z}$ at the final loading step: section by plane Orz (a); threedimensional state at the final stage of loading (b)

Thus, the presented calculation scheme with the loading steps by internal pressure $P$, taking into account the formation of the contact area of the elastic seals and the borehole wall, allows us to describe the pressure distribution at the "seal - rock" boundary, as well as to study the stress-strain state of the geomedium at a given working pressure in the packer depending on the distance between the seals.

\section{Conclusions}

The problems of implementing an innovative method of intensifying the processes of coal seams underground degassing by interval hydraulic fracturing are considered. As a result of experimental and theoretical studies, numerical studies have been performed that determine the possibility of the development of an axisymmetric hydraulic fracture in an isotropic medium from a well using the created equipment. The developed numerical scheme allows to study the development of hydraulic fractures cracks in an elastic medium. The results obtained show a significant effect of the internal pressure and its effect of the change on the fracture path.

The results can be used in the selection of parameters and technical means of coal seams hydraulic fracturing for work on the intensification of the process of degassing from underground mine workings through degassing open-hole wells.

\section{Acknowledgement}

This study was supported by the Russian Science Foundation, Project No. 17-17-01143.

\section{References}

1. K. N. Trubetskoy, A. D. Ruban, V. S. Zaburdyaev, J Min Sci 47(1), 1-9 (2011) 
2. K. N. Trubetskoy, V. V. Gurianov, Coal, 9, 3-6 (2003)

3. A. D. Ruban, V. S. Zaburdyaev, G. S. Zaburdyaev, N. G. Matvienko, Methane in coal mines and mine camps of Russia: forecast, extraction, and use (Publ. house ICEMR, Moscow, 2006)

4. N. V. Nozhkin, Advance degassing of coal deposits (Nedra, Moscow, 1979)

5. I. V. Sergeev, V. S. Zaburdyaev, Management of gas release in coal mines during coal face operations (Nedra, Moscow, 1992)

6. S. V. Slastunov, Advance degassing and extraction of methane from coal deposits (Publ. house MSMU, Moscow, 1996)

7. Yu. N. Malyshev, Yu. L. Hudin, M. P. Vasilchuk, Problems of developing methanebearing coal seams in the Kuznetsk coal basin (Publ. house Academy of Mining Sciences, Moscow, 1997)

8. L. A. Puchkov, S. V. Slastunov, B. I. Fedunets, Prospects for methane production in the Pechora coal basin (Publ. house MSMU, Moscow, 2004)

9. L. A. Puchkov, S. V. Slastunov, G. M. Present, Mining Informational and Analytics Bulletin 6, 6-10 (2002)

10. V. I. Klishin, D. I. Kokoulin, B. Kubanychbek, M. K. Durnin, Coal 4, 40-42 (2010)

11. V. I. Klishin, G. Yu. Opruk, A. L. Tatsienko, IOP Conference Series: Earth and Environmental Science 53(1), 012019 (2017)

12. S. G. Cherny, V. N. Lapin, D. V. Esipov, D. S. Kuranakov, Methods for modeling crack nucleation and propagation (Publ. house SBRAS, Novosibirsk, 2016)

13. F. Jun, D. Linming, H. Hua, D. Taotao, Z. Shibin, G. Bing, S. Xinglin, Int J Min Sci \& Tech 22(2), 177-181 (2015)

14. J. Deng, Q. Yang, Y. Liu, Yi Liu, G. Zhang, Comput Geotech 94, 118-133 (2018)

15. M. V. Kurlenya, S. V. Serdyukov, A. V. Patutin, T. V. Shilova, J Min Sci 53(6), 975980 (2017)

16. R. Jeffrey, K. Mills, X. Zhang, Proc. $3^{\text {rd }}$ Int Workshop on Mine Hazards Prevention and Control (AMS, Brisbane, 2013)

17. V. I. Klishin, O. V. Taylakov, G. Yu. Opruk, M. P. Makeev, S. V. Sokolov, A. S. Teleguz, A. L. Tatsienko, IOP Conference Series: Earth and Environmental Science 377(1), 012034 (2019)

18. El Rabaa W., Experimental study of hydraulic fracture geometry initiated from horizontal wells (SPE Annual Technical Conference and Exhibition, Abu-Dabi, 1989)

19. V. I. Klishin, D. I. Kokoulin, B. Kubanychbek, A. P. Gurtenko, J Min Sci 46(4), 411415 (2010)

20. V. I. Klishin, G. Yu. Opruk, D. I. Kokoulin, A. L. Tatsienko, IOP Conference Series: Earth and Environmental Science 262(1), 012028 (2019)

21. A. L. Tatsienko, S. V. Klishin, Mining Informational and Analytics Bulletin 11(49), 4957 (2018) 